\title{
Posterior spinal artery aneurysm as an unlikely culprit for perimesencephalic pattern subarachnoid hemorrhage: illustrative case
}

\author{
Armaan K. Malhotra, MD, ${ }^{1}$ Jerry C. Ku, MD, ${ }^{1}$ Vitor M. Pereira, MD, MSc, ${ }^{2}$ and Ivan Radovanovic, MD, ${ }^{2} D^{3}$ \\ ${ }^{1}$ Division of Neurosurgery, Department of Surgery; ${ }^{2}$ Division of Interventional Neuroradiology, Department of Medical Imaging, University of Toronto, Toronto, Ontario, Canada; and \\ ${ }^{3}$ Division of Neurosurgery, Toronto Western Hospital, Toronto, Ontario, Canada
}

\begin{abstract}
BACKGROUND Angiogram-negative nontraumatic subarachnoid hemorrhage (SAH) can be diagnostically challenging, and a broad differential diagnosis must be considered. Particular attention to initial radiographic hemorrhage distribution is essential to guide adjunctive investigations. Posterior spinal artery aneurysms are rare clinical entities with few reported cases in the literature. An understanding of isolated spinal artery aneurysm natural history, diagnosis, and management is evolving as more cases are identified.

OBSERVATIONS Isolated thoracic posterior spinal artery aneurysm can be the culprit lesion in perimesencephalic distribution SAH. Embolization resulted in complete aneurysm occlusion and did not result in periprocedural morbidity. At the 1-year follow-up, the patient was neurologically intact with no recurrence on magnetic resonance angiography.
\end{abstract}

LESSONS This case report highlighted the presentation, diagnostic workup, clinical decision-making, and endovascular intervention for a woman who presented with SAH secondary to posterior spinal artery aneurysm. After initially negative results on vascular imaging, dedicated spinal vascular imaging revealed the location of the aneurysm. Multiple treatment modalities exist for isolated spinal artery aneurysms and must be selected on the basis of patient- and lesion-specific characteristics.

https://thejns.org/doi/abs/10.3171/CASE21103

KEYWORDS case report; posterior spinal artery aneurysm; spinal subarachnoid hemorrhage; perimesencephalic; spinal aneurysm

Catheter angiogram-negative subarachnoid hemorrhage (SAH) poses a diagnostic and therapeutic challenge. Failure to detect underlying lesions can result in significant morbidity and death associated with rebleeding. ${ }^{1,2}$ Approximately $10 \%$ to $20 \%$ of SAH cases are diagnostic angiogram negative; subsequent discovery of a culprit lesion occurs in $2 \%$ to $21 \%$ of cases. ${ }^{2}$ Specifically, the pattern of hemorrhage should be classified as perimesencephalic or non-perimesencephalic because the likelihood of finding a culprit lesion differs significantly between the two. ${ }^{1,2} \mathrm{SAH}$ attributable to spinal vascular etiologies such as aneurysms occurs in less than $1 \%$ of SAH cases. ${ }^{3}$ The incidence of spinal artery aneurysms has been shown to be 1 in more than 3,000 angiograms. ${ }^{4}$ Spinal artery aneurysms are commonly flow-related lesions associated with arteriovenous malformations and, less frequently, dural arteriovenous fistulas. ${ }^{2}$ Other etiologies include bilateral vertebral artery occlusion, coarctation of the aorta, and moyamoya disease. ${ }^{2}$ Another cluster of spinal aneurysms has been noted in patients with underlying conditions that affect vascular wall integrity, such as vasculitis, infectious etiologies, or connective tissue disorders. ${ }^{5}$ Isolated spinal artery aneurysms are infrequent and not well understood.

A ruptured isolated posterior spinal artery (PSA) aneurysm as an etiology for a catheter angiogram-negative SAH is a rare clinical entity with few documented cases to date. Herein we describe a case of an isolated PSA aneurysmal SAH with emphasis on diagnostic workup, imaging investigations, and management nuances, including surgical and endovascular decision-making. Furthermore, we review isolated PSA aneurysm cases treated with embolization.

\section{Illustrative Case}

A 76-year-old woman presented to urgent care with a necrotizing soft tissue infection of her right arm and fourth digit. She had a history

ABBREVIATIONS ATECO = auto-triggered elliptic centric ordered; $C T$ = computed tomography; ICG = indocyanine green; $\mathrm{MRI}=$ magnetic resonance imaging; $\mathrm{NBCA}=\mathrm{N}$-butyl cyanoacrylate; $\mathrm{PSA}=$ posterior spinal artery; $\mathrm{SAH}=$ subarachnoid hemorrhage .

INCLUDE WHEN CITING Published May 31, 2021; DOI: 10.3171/CASE21103.

SUBMITTED February 15, 2021. ACCEPTED March 8, 2021.

(c) 2021 The authors, CC BY-NC-ND 4.0 (http://creativecommons.org/licenses/by-nc-nd/4.0/). 
of nail biting but no history of injection drug use, penetrating foreign body injury, or trauma. Her medical history was significant for ischemic optic neuropathy, hypothyroidism, dyslipidemia, prior temporal arteritis, and hypertension. She underwent operative debridement of the necrotizing infection and amputation of her fourth digit. She was monitored closely in the intensive care unit, where she was maintained on broadspectrum antibiotics. Her intraoperative tissue cultures speciated group A Streptococcus, and her therapy was narrowed accordingly.

During her hospitalization, she voiced complaints of lower back pain. Approximately 10 days after her admission, she developed sudden onset of headache, hypertension, severe nuchal rigidity, and depressed level of consciousness. Computed tomography (CT) imaging of her head demonstrated what was initially suspected to represent perimesencephalic pattern $\mathrm{SAH}$ (Fig. 1A). There was discussion that the volume of blood product in the prepontine cistern and surrounding the upper cervical cord was thicker than expected in true perimesencephalic pattern bleeds (Fig. 1B). CT angiography did not reveal a culprit lesion. Subsequent digital subtraction angiography of the intracranial vasculature was negative for any vascular pathology. Given the extensive upper cervical hemorrhage in the absence of a culprit vascular lesion on formal angiography, spinal vascular magnetic resonance imaging (MRI) with auto-triggered elliptic centric ordered (ATECO) sequence was performed. It revealed extensive spinal subarachnoid blood with a well-defined dorsally located $7 \times 4-\mathrm{mm}$ lesion in the intradural extramedullary compartment most consistent with a PSA aneurysm at T10-11 (Fig. 1C and D).
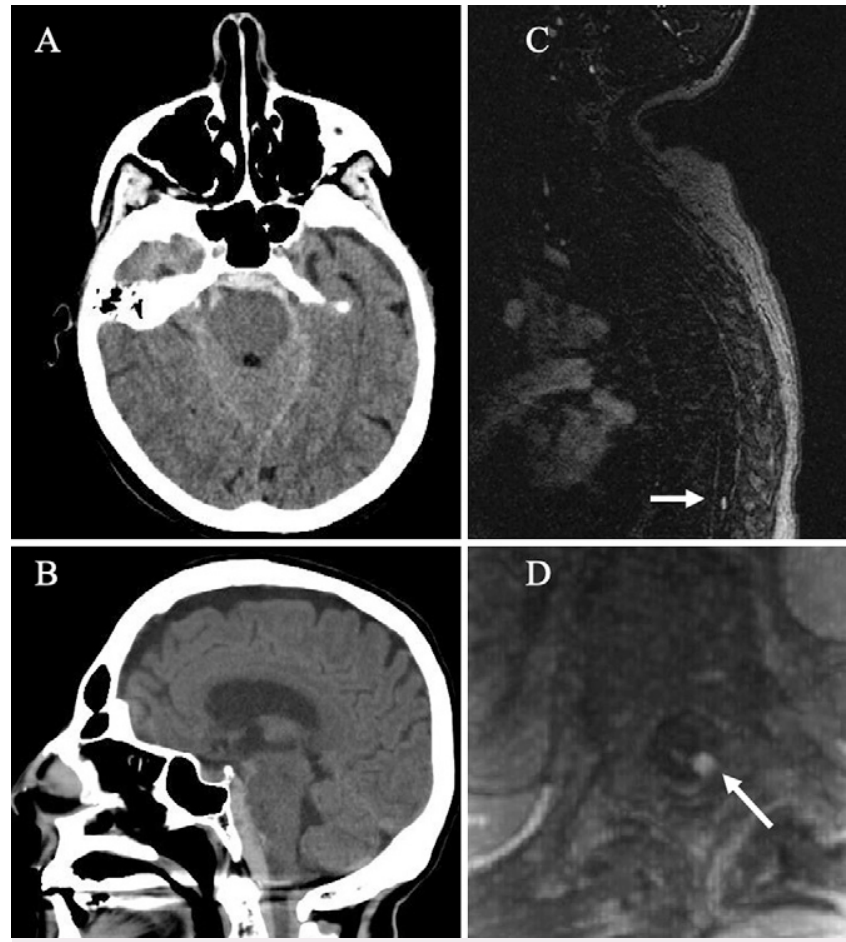

FIG. 1. A and B: Axial and sagittal noncontrast CT demonstrating an atypical perimesencephalic pattern $\mathrm{SAH}$ with extension to the cervical spinal cord. C and D: Magnetic resonance angiography, sagittal and axial cuts, highlighting the left-sided posterior intradural extramedullary enhancing lesion suspicious for PSA aneurysm (white arrows).
The patient then underwent spinal angiography. Injection through the left T11 segmental artery showed a fusiform dilatation of the radiculopial PSA consistent with a dissecting aneurysm (Fig. 2A and B). The artery of Adamkiewicz was supplied by the L1 segmental artery; therefore, proceeding with embolization in the same procedure was believed to be safe. Multidisciplinary discussion with endovascular and open neurovascular experts concluded that endovascular embolization was the safest option given the configuration of the aneurysm. A C2 Cobra Glidecath catheter (Terumo) was used as a guide catheter to cannulate the segmental artery at T11 for stability. A 1.20-Fr Magic microcatheter (Balt) was then used with the aid of a microwire to enter the PSA that directly fed the aneurysm. Embolization using 50\% Lipidol and N-butyl cyanoacrylate (NBCA) resulted in complete aneurysm occlusion (Fig. 2C). The artery of Adamkiewicz was patent at the end of the procedure.

Postoperatively, spinal MRI ATECO showed no evidence of residual aneurysm. The patient's course was complicated by transient dysarthria, right arm weakness, and seizures. Repeat CT angiography showed severe anterior and posterior circulation vasospasm. Small cortical and subcortical strokes were demonstrated in the left centrum semiovale and temporoparietal cortex. Her condition was managed successfully with blood pressure augmentation. Blood culture results were never positive during her admission, although the occurrence of a septic aneurysm was possible given her initial necrotizing soft tissue infection. After supportive treatment for her $\mathrm{SAH}$, the patient was discharged to rehabilitation.

At the 1-year follow-up, MRI ATECO was negative for any recurrence of the embolized PSA aneurysm. Clinically, the patient was walking independently with mildly impaired tandem gait and had normal cognitive function.

\section{Discussion}

We describe a clinical case of spontaneous $\mathrm{SAH}$ in a patient hospitalized for necrotizing fasciitis. Although initially reported as a perimesencephalic pattern hemorrhage, the excessive volume of
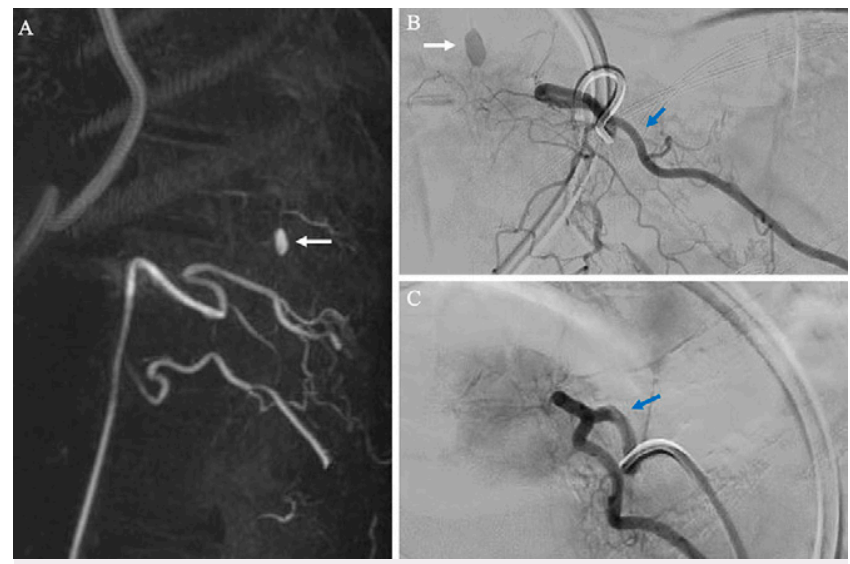

FIG. 2. A: Spinal angiogram multiframe spin image demonstrating filling of ovoid $7 \times 4-\mathrm{mm}$ dissecting PSA aneurysm (white arrow). $B$ and C: Microcatheter injection of the left T11 segmental artery (blue arrows) before and after embolization runs demonstrating successful obliteration of the aneurysm (white arrow). 
blood surrounding the brainstem and upper cervical cord prompted additional investigation with spinal vascular imaging, which revealed a ruptured thoracic PSA aneurysm, an exceedingly rare diagnosis. The aneurysm was treated successfully with endovascular embolization with good clinical and radiological outcomes.

\section{Observations}

The etiology of isolated PSA aneurysms is uncertain. Our patient did not have clear flow-influencing factors such as arteriovenous malformation, bilateral vertebral artery occlusion, or coarctation of the aorta. Other mechanisms include vasculitis or infectious processes that compromise the integrity of the vessel wall. Despite the absence of positive blood culture results, there was a strong temporal relationship between her hemorrhage and her presentation with necrotizing fasciitis. The possibility of an infectious or mycotic etiology behind the aneurysm was raised, which could not be confirmed because no histopathological review was possible. In one review of spinal artery aneurysmal SAH, 2 cases were suspected related to infectious etiologies, including syphilis and systemic candidiasis. ${ }^{2,6,7}$ The driving mechanism of isolated PSA aneurysms is unclear. Histopathological reports of isolated PSA aneurysmal specimens have shown dissection patterns in the vessel wall involving the tunica media as the mechanism of rupture often leading to a fusiform appearance as opposed to a well-defined neck. $^{8-10}$ This pathophysiology can have important implications for surgical approach because clipping may not be as feasible for intracranial aneurysms that have predilection for occurrence at branching points. Given the small caliber of offending vessels in this region, several reports have also described spontaneous thrombosis and regression of these aneurysms. ${ }^{10-13}$

Taken together, approximately 30 cases in the literature have documented ruptured posterior axis spinal artery aneurysms diagnosed angiographically. ${ }^{10,12}$ More anterior axis spinal aneurysms have been described and appear to be related more commonly to systemic vasculitis or infectious etiologies. ${ }^{3}$ Clinical characteristics at presentation are variable. Sudden severe back pain mimicking aortic dissection is common at presentation and may be associated with lower extremity weakness, paresthesia, and urinary incontinence. ${ }^{14}$ The quality of pain has been described as subacute onset and may be radicular, mechanical, or persistent at rest. ${ }^{11,13}$ Hypertensive crisis and thunderclap headache and cranial neuropathies are common presenting features. ${ }^{13-15}$ Initial presentation with thunderclap headache and nuchal rigidity followed by acute onset of paraplegia has also been described. ${ }^{16}$ This was the case with our patient because the presentation was a more classic thunder clap headache with depressed level of consciousness, prompting neuroimaging.

One of the largest groupings of anterior and PSA aneurysm cases was documented by Geibprasert et al. ${ }^{3}$ In their cohort, 11 of 32 cases were associated with either paraparesis/paraplegia or tetraparesis/tetraplegia, 21 had $\mathrm{SAH}$ at presentation, and 1 had low back pain with sciatica. At presentation, one study showed that among 9 noncontrast CT head scans performed, subarachnoid blood was visualized in 5 cases, of which 3 were centered in the prepontine cistern and 2 were in the convexity. ${ }^{14}$ The clinical features at presentation are highly variable and emphasize the importance of a broad differential diagnosis. In patients with localization of pain or neurological deficit to the spinal cord, dedicated imaging is clearly indicated.
In the workup of all patients with angiogram-negative SAH, however, the indications for dedicated spinal imaging are much less clear. Little et al. found the yield of MRI of the cervical and thoracolumbar spine to be $3 \%$ and $0 \%$, respectively, in classic pattern angiogram-negative $\mathrm{SAH}$ and $0 \%$ and $0 \%$, respectively, in perimesencephalic pattern $\mathrm{SAH}^{2}$ Additionally, other studies have found similarly low rates detecting a bleeding source with MRI of the cervical spine. ${ }^{17,18}$ In our patient, the pattern of bleeding was centered around the upper cervical cord, prompting full spinal vascular imaging. In similar circumstances of angiogram-negative SAH that do fit the strict criteria of perimesencephalic pattern $\mathrm{SAH}$, it is recommended that additional imaging be pursued on the basis of specific clinical and radiological findings.

Management of PSA aneurysms can be broadly considered as surgical, endovascular, and conservative. The goals of surgical and endovascular interventions are to reduce the risk of rebleeding, which has been reported previously with significant neurological morbidity and mortality. ${ }^{18,19}$ Some authors have described conservative management, citing the observed tendency for thrombosis and subsequent collapse of aneurysms in this location. ${ }^{15}$ They advocate a waitand-watch approach, suggesting repeat angiography 7 to 10 days after initial diagnosis to compare aneurysm growth, stability, or regression before intervention or decision for continued observation. ${ }^{15}$ Furthermore, anterior location can confer a more challenging operative approach and higher procedural risk of complications related to a periprocedural infarct. Based on the small number of reported cases, it is difficult to make claims regarding the natural history of these aneurysms; however, given the risk of severe morbidity associated with rebleeding, we advocate for prompt intervention to secure ruptured isolated spinal artery aneurysms when possible.

Renieri et al. performed a multicenter retrospective review of spinal artery aneurysms and reported on 11 cases, of which 3 were radiculomedullary and 8 were radiculopial. ${ }^{14}$ In the radiculomedullary cohort, 1 case was managed conservatively because of anatomical concerns and proximity to anterior spinal artery origin, 1 was managed with open surgical trapping, and 1 was managed with endovascular coiling. Within the radiculopial group, 4 cases were managed through endovascular means with coil or particulate embolization of the parent radicular artery. Three cases were managed conservatively, whereas 1 was managed surgically. In this cohort, 7 of 8 patients with available follow-up were functionally independent 1 month after discharge, and no deaths were documented.

On the other hand, in a large review of PSA aneurysmal SAH performed by lkeda et al. that identified 19 cases, there was a significant tendency toward open surgical management. ${ }^{13}$ In their study, 12 cases were managed with open surgery, 4 with an endovascular approach, and 3 with a conservative approach. Outcomes were reported as stable in 4 cases and improved in 14 cases, and there was 1 death associated with conservative management and rebleeding. ${ }^{19}$ Based on the available literature, it appears that functional outcome is favorable in patients undergoing either endovascular or surgical intervention, although sufficient numbers for meaningful statistical inferences are not available. The treatment-related morbidity in surgical and endovascular cohorts is difficult to compare, and the rates of sustained aneurysm obliteration at long-term follow-up are uniformly high. The finding of vasospasm, as seen in our patient, is not commonly reported in ruptured spinal artery aneurysms and likely reflects the large volume of posterior fossa blood product, as evident on presentation (Fig. 1A and B). 
Surgical management has been the mainstay modality among reported cases. ${ }^{3,8,11,13-16} \mathrm{~A}$ common approach is hemilaminectomy and clipping or parent vessel sacrifice given the dorsolateral loca-

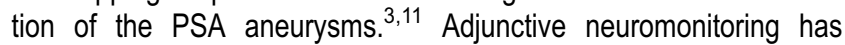
been described in addition to the use of intraoperative indocyanine green (ICG), although in cases with thrombosed aneurysms at the time of surgery, ICG has limited utility. ${ }^{11,13,20}$ Furthermore, 2 cases were found to have spontaneously regressed either through parent vessel thrombosis or lack of aneurysm at surgery. ${ }^{6,21}$ If anatomical restrictions preclude clipping or the lesion is closely associated with the artery of Adamkiewicz, the anterior spinal artery, or the PSA and the risk of periprocedural cord infarct is high, wrapping aneurysms with muslin or direct vessel reconstruction has been described previously. $8,22,23$

Endovascular intervention may be broadly classified into coiling and particle embolization. Given the proclivity of isolated PSA aneurysms to result from dissection, most described cases are fusiform as opposed to saccular with a well-defined neck, making them unfavorable for coiling. Some groups discuss direct coiling of radiculopial arteries for intended vessel sacrifice. Renieri et al. identify the theoretical risk of aneurysm reconstitution through anastamotic vessel networks and suggest particle embolization to reduce the risk of impacting distal collateral circulation. ${ }^{14}$ The same group cautioned against the use of particle embolization in proximity to the subpial conus basket given the dense arterial network at that level. In addition to previously mentioned endovascular management strategies, Simon-Gabriel et al. describe a case of ruptured fusiform craniocervical anterior spinal artery aneurysm treated successfully with flow diversion of the parent vertebral artery. ${ }^{24}$ In our patient, angiography revealed the artery of Adamkiewicz originating from the L1 segmental artery, whereas the aneurysm originated from T11. We therefore proceeded with embolization using 50\% Lipidol and NBCA. The latter forms rapid polymers in vivo with good tissue adherence, and Lipidol acts as a radio-opaque vehicle as well as a polymerization retardant. ${ }^{25}$ With continued development in endovascular technology and microcatheters, selective cannulization of segmental spinal arteries and directed microcatheter placement for embolization of PSA aneurysms are technically feasible.

At our institution, these cases are typically reviewed at multidisciplinary rounds attended by both endovascular and neurosurgical experts. In this situation, the anatomical location and lack of vessel tortuosity were favorable for an endovascular approach. Furthermore, given the recent necrotizing infection and multiple skin grafts, endovascular embolization was believed to be lower risk than a prone position for laminectomy and clipping. In this case, endovascular treatment resulted in aneurysm obliteration with good neurological outcome, no evidence of spinal cord infarct or neurological morbidity, and a sustained effect evident at the 1-year follow-up.

\section{Lessons}

We describe the case of a 76-year-old woman who presented initially with necrotizing soft tissue infection followed by SAH attributable to a ruptured radiculopial PSA dissecting aneurysm. Additional spinal vascular imaging for angiogram-negative SAH should be sought in specific circumstances, such as high blood volume on CT surrounding the brainstem or upper cervical cord, so as not to miss any possible culprit lesions. The patient was successfully treated using endovascular glue embolization with an independent functional outcome and sustained aneurysm occlusion that persisted at the long-term 1-year follow-up. Furthermore, by presenting a review of the management philosophy for PSA aneurysms with discussion of surgical and endovascular treatment modalities, we demonstrate that both are acceptable treatment options for this relatively rare clinical entity.

\section{References}

1. Canhão P, Ferro JM, Pinto AN, et al. Perimesencephalic and nonperimesencephalic subarachnoid haemorrhages with negative angiograms. Acta Neurochir (Wien). 1995;132(1-3):14-19.

2. Little AS, Garrett M, Germain R, et al. Evaluation of patients with spontaneous subarachnoid hemorrhage and negative angiography. Neurosurgery. 2007;61(6):1139-1151.

3. Geibprasert S, Krings T, Apitzsch J, et al. Subarachnoid hemorrhage following posterior spinal artery aneurysm. A case report and review of the literature. Interv Neuroradiol. 2010;16(2):183-190.

4. Djindjian R. Angiography of the spinal cord. Surg Neurol. 1974;2(3): 179-185.

5. Ronchetti G, Morales-Valero SF, Lanzino G, Wald JT. A cause of atypical intracranial subarachnoid hemorrhage: posterior spinal artery aneurysms. Neurocrit Care. 2015;22(2):299-305.

6. Berlis A, Scheufler KM, Schmahl C, et al. Solitary spinal artery aneurysms as a rare source of spinal subarachnoid hemorrhage: potential etiology and treatment strategy. AJNR Am J Neuroradiol. 2005;26(2):405-410.

7. Rengachary SS, Duke DA, Tsai FY, Kragel PJ. Spinal arterial aneurysm: case report. Neurosurgery. 1993;33(1):125-130.

8. Massand MG, Wallace RC, Gonzalez LF, et al. Subarachnoid hemorrhage due to isolated spinal artery aneurysm in four patients. AJNR Am J Neuroradiol. 2005;26(9):2415-2419.

9. Nemecek AN, Sviri G, Hevner R, et al. Dissecting aneurysm of the thoracic posterior spinal artery. Case illustration. J Neurosurg Spine. 2006;5(6):555.

10. Sung TH, Leung WK, Lai BM, Khoo JL. Isolated spinal artery aneurysm: a rare culprit of subarachnoid haemorrhage. Hong Kong Med J. 2015;21(2):179-182.

11. Takebayashi K, Ishikawa T, Murakami M, et al. Isolated posterior spinal artery aneurysm presenting with spontaneous thrombosis after subarachnoid hemorrhage. World Neurosurg. 2020;134:544-547.

12. Sato K, Roccatagliata L, Depuydt S, Rodesch G. Multiple aneurysms of thoracic spinal cord arteries presenting with spinal infarction and subarachnoid hemorrhage: case report and literature review. Neurosurgery. 2012;71(5):E1053-E1058.

13. Ikeda S, Takai K, Kikkawa Y, et al. Ruptured posterior spinal artery aneurysm: intraoperative and histologic findings with appreciable thrombosis. Spine J. 2016;16(3):e215-e217.

14. Renieri L, Raz E, Lanzino G, et al. Spinal artery aneurysms: clinical presentation, radiological findings and outcome. J Neurointerv Surg. 2018;10(7):644-648.

15. van Es AC, Brouwer PA, Willems PW. Management considerations in ruptured isolated radiculopial artery aneurysms. A report of two cases and literature review. Interv Neuroradiol. 2013;19(1):60-66.

16. Cadieux M, Tso M, Fox S, Jacobs WB. Spontaneous spinal subarachnoid hemorrhage from a ruptured radiculopial artery aneurysm. World Neurosurg. 2021;145:114-118.

17. Sadigh G, Holder CA, Switchenko JM, et al. Is there added value in obtaining cervical spine MRI in the assessment of nontraumatic angiographically negative subarachnoid hemorrhage? A retrospective study and meta-analysis of the literature. J Neurosurg. 2018; 129(3):670-676.

18. Maslehaty H, Petridis AK, Barth $H$, Mehdorn HM. Diagnostic value of magnetic resonance imaging in perimesencephalic and nonperimesencephalic subarachnoid hemorrhage of unknown origin. $J$ Neurosurg. 2011;114(4):1003-1007. 
19. Koçak A, Ateş O, Cayli SR, Saraç K. Isolated posterior spinal artery aneurysm. Br J Neurosurg. 2006;20(4):241-244.

20. Takata M, Takayama M, Yokoyama Y, et al. An isolated posterior spinal aneurysm resection in which intraoperative electrophysiological monitoring was successfully used to locate the lesion and to detect the possibility of ischemic complications. Spine (Phila $\mathrm{Pa}$ 1976). 2016;41(1):E46-E49.

21. Goto Y, Kamijyo Y, Yonekawa Y, Kikuchi H. Ruptured aneurysm of the posterior spinal artery of the upper cervical spinal cord: case report. Neurosurgery. 1988;22(3):558-560.

22. Taniura $S$, Watanebe T. A ruptured dissecting aneurysm of the anterior radiculomedullary artery caused by vertebral angiography. Neuroradiology. 2000;42(7):539-542.

23. Vishteh AG, Brown AP, Spetzler RF. Aneurysm of the intradural artery of Adamkiewicz treated with muslin wrapping: technical case report. Neurosurgery. 1997;40(1):207-209.

24. Simon-Gabriel CP, Urbach H, Meckel S. Ruptured fusiform aneurysm of the anterior spinal artery: successful treatment with flow diverter stent placed in the feeding vertebral artery. Clin Neuroradiol. 2018;28(4):613-616.
25. Brothers MF, Kaufmann JC, Fox AJ, Deveikis JP. n-Butyl 2-cyanoacrylate-substitute for IBCA in interventional neuroradiology: histopathologic and polymerization time studies. AJNR Am J Neuroradiol. 1989;10(4):777-786.

\section{Disclosures}

The authors report no conflict of interest concerning the materials or methods used in this study or the findings specified in this paper.

\section{Author Contributions}

Conception and design: Malhotra, Ku. Acquisition of data: Malhotra, Pereira. Analysis and interpretation of data: Malhotra, Ku. Drafting the article: Malhotra, Ku, Pereira. Critically revising the article: Malhotra, $\mathrm{Ku}$, Radovanovic. Reviewed submitted version of manuscript: Malhotra, Pereira, Radovanovic. Study supervision: Pereira, Radovanovic.

\section{Correspondence}

Armaan K. Malhotra: University of Toronto, Toronto, ON, Canada. ak.malhotra@mail.utoronto.ca. 